\title{
Adaptación y validación de una escala para la evaluación del desempeño profesional del entrenador de fútbol en base a su formación permanente, nivel de TIC y autoevaluación \\ Adaptation and validation of a scale for the evaluation of the professional performance of the football trainer based on his/ her continuous training, level of ICT and self-evaluation

\author{
*Ignacio Ballester Esteve, *Rocío Fernández Piqueras, **David Parra-Camacho \\ *U niversidad Católica deValencia San Vicente M ártir (España), **U niversidad deValencia (España)
}

\begin{abstract}
Resumen. La presente investigación tiene por objetivo adaptar y validar una escala para la evaluación del desempeño profesional de los entrenadores de fútbol basada en su formación previa y en el nivel de competencias digitales que posea dicho entrenador (Escala para la evaluación del desempeño profesional del entrenador de fútbol en base a su formación permanente, nivel deTIC y autoevaluación). La muestra del estudio está constituida por un total de 412 entrenadores en formación, de los cuales el $91.7 \%$ son hombres y el $8,3 \%$ restante son mujeres. Todos estos sujetos son alumnos de los cursos de formación de entrenadores de fútbol de la Federación de Fútbol de laComunidadValenciana(FFCV), distribuidos por los diferentes niveles técnicos, aunque el más representado es el UEFA C con un $71.1 \%$. El diseño de la escala se realizó a partir del cuestionario realizado por Zambrano, Meda y Lara (2005). Se realizó un análisis factorial exploratorio (AFE) y un análisis factorial confirmatorio (AFC), que permitieron identificar tresfactores en los quese agruparon losindicadores: interéspor laformación continua ( 4 ítems), formación en TIC ( 3 ítems) y autoevaluación (4 ítems). Los diferentes indicadores de bondad de ajuste mostraron valores adecuados: $\left(x^{2} / g \mid=2.77\right) ;$ RMSEA $=.057$ (Intervalo de confianza=.042-.072); $C F|=.97 ;| F \mid=.97$. Se comprobó la fiabilidad mediante los valores de fiabilidad compuesta, alfa de Cronbach y la varianza media extraída. Los resultados de la investigación sostienen la fiabilidad y validez de la escala para valorar el desempeño profesional de los entrenadores de fútbol.
\end{abstract}

Palabras Clave: entrenadores de fútbol, desempeño profesional, TIC, formación continua, autoevaluación.

\begin{abstract}
This research aims to adapt and validate a scale for the eval uation of the professional performance of football coaches based on their previous training and the level of digital competencies that the coach possesses (Scale for the evaluation of the professional performance of football coaches based on their permanent training, level of ICT and self-eval uation). The study sample is made up of atotal of 412 coaches in training, of which $91.7 \%$ are men and the remaining $8.3 \%$ are women. All of these subjects are students of the training courses for football coaches of the Valencian Football Federation (FFCV), distributed by the different technical levels, although the most represented is UEFA C with 71.1\%. The design of the scale was based on the questionnaire carried out by Zambrano, M eda and Lara (2005). An exploratory factorial analysis (EFA) and a confirmatory factorial analysis (CFA) were carried out, which allowed identifying three factors in which the indicators were grouped: interest in continuoustraining ( 4 items), ICT training ( 3 items) and self-evaluation ( 4 items). The different goodnessof-fit indicators showed adequate values: $\left(\mathrm{x}^{2} / \mathrm{gl}=2.77\right) ; \mathrm{RMSEA}=.057$ (Confidence Interval=.042-.072); CFI=.97; IFI=.97. Reliability was checked using the composite reliability values, Cronbach's al phaand the average variance extracted. The results of the research support the reliability and validity of the scale to assess the professional performance of soccer coaches.
\end{abstract}

Keywords: football coaches, professional performance, ICT, continuous training, self-evaluation.

\section{Introducción}

En el año 2015, la U nion of European Football Asociation (en adelante UEFA) celebró una convención con el objetivo de realizar una profunda reflexión en relación con las titulaciones técnicas (UEFA, 2015). Entre sus

Fecharecepción: 10-09-20. Fechadeaceptación: 03-11-20

David Parra-Camacho

david.parra-camacho@uv.es conclusiones más importantes, destacó la necesidad de realizar un cambio metodológico en la gestión de los cursos formativos, especial mente en la labor como docentes de los entrenadores. Se realizaron, por una parte, modificaciones importantes en las asignaturas, destacando la nueva distribución de aquellas materias que demandaban una actualización debido principalmente a la evolución natural de las sociedades actuales, muy condicionadas por el continuo desarrollo de las tecnologías de la información y comunicación (en adelante TIC). 
Por otra parte, se puso de manifiesto la necesidad de un giro pedagógico en la enseñanza del fútbol en todos los niveles. Estas mismas demandas fueron ajustadas en la formación universitaria, acorde al Espacio Europeo de Educación Superior (EEES), donde la formación didáctica de los especial istas deportivos se adaptó a la nueva situación metodológica y social. Algunas de las propuestas deportivas universitarias se realizaron a través de simulaciones para la mejora del desempeño profesional, como demostró la investigación de Rojas, Cepero, Zurita, \& Chinchilla (2015).

Si se analiza la evolución en los planes de estudios de los entrenadores de fútbol, puede observarse cómo han ido aumentando las horas en aquellas materias vincula das a contenidos TIC y en aquellas sobre didáctica y metodología de la enseñanza del fútbol, como Filosofía Didáctica, Periodización y Sesiones de entrenamiento, en detrimento de otras más tradicionales como son las de técnica individual. Hasta la convención de la UEFA (2015), la asignatura de Nuevas Tecnologías, se impartía en los cursos de nivel I, II y III, pero solo alcanzaba un número significativo de horas en el último curso, es decir en el III. A partir de dicho curso 2015, la asignatura de NuevasTecnologías, pasó a denominarse, Análisis, además, la materia aumentó considerablemente de horas, realizado de forma progresiva. Así, la asignatura de Análisis ha visto aumentada sus horas desde el primer curso hasta el último, siendo en el nivel profesional una materia con una carga de 50 horas, es decir, la disciplina que cursa el mayor número de horas junto con la asignatura de Sesiones.

Respecto al cambio metodológico, éste ha sido objeto de discusión en investigaciones previas, como la realizada por Lledó, M artínez y Huertas (2014). En dicha investigación se manifestaba una diferencia de recursos docentes entre los técnicos formados en contenidos exclusivamente deportivos, con un escaso bagaje a nivel docente, los cuales, empleaban metodologías ana líticas y tradicionales frente a los entrenadores que ha bían estudiado Educación Física, que mostraban competencias docentes y didácticas muy superiores que el colectivo anterior. El motivo de la investigación se justificaba por la capital importancia de los entrenadores en el desarrollo de la personalidad y en la formación de los jóvenes deportistas en edad de iniciación. En este sentido, en el proceso de evaluación metodológico en ámbitos particulares como el de profesorado, deportistas 0 el de los técnicos deportivos, produce un aumento de la tensión en el sujeto por sentirse evaluado. Este aspecto, genera cambios entre los implicados y en muchos casos modifica su conducta, la cual puede condicionar los resultados obtenidos (González, Campos, \& Romero, 2014). Por otro lado, en la evaluación de los técnicos deportivos, en muchas ocasiones son sus deportistas, los queevalúan al entrenador. ParaFraile-Aranda, deDiegoVallejo y Boada (2011), en su caso con jugadores de ba Ioncesto y balonmano, muestran una preferencia hacia entrenadores que se acogen a modelos metodológicos más dialogadores, críticos e innovadores frente aquellos que se sitúan en los modelos más técnicos.

Debido a estas modificaciones realizadas en la formación del entrenador, aumento de horas en contenidosTIC y cambios pedagógicos, un razonamiento a plantearse consiste en estudiar si el desempeño profesional de los nuevos entrenadores se ha visto aumentado. El desempeño profesional es un constructo estudiado en diferentes contextos profesionales, como el educativo, pero poco analizado en el contexto deportivo, especialmente en el de los entrenadores deportivos de fútbol (Marcelo, 2001). La evaluación del desempeño profesional debe siempre orientar y promover la calidad de la formación, especialmente en la mejora del desarrollo profesional, buscando como última finalidad garantizar el perfeccionamiento del educador (en nuestro caso también entrenador) en el contexto donde realice su trabajo (Arias de Vega \& O Imos, 2015). En el ámbito educativo, destaca especialmente en la literatura las investigaciones centradas en el periodo universitario. Así pues, el desempeño profesional, es un constructo que establece los niveles de competencia de las personas implicadas directamente en un contexto y para ello, la evaluación de su desempeño profesional demuestra, un mayor nivel de competencia. Para Arias deVega y $0 \mathrm{I}-$ mos (2015), la importancia de la evaluación del desempeño profesional entre el profesorado es importante para poder determinar los niveles de efectividad en el desarrollo de las competencias educativas.

Siguiendo en el ámbito educativo universitario, Zambrano et al. (2005), centran la evaluación del desempeño profesional, en el cumplimiento de los objetivos docentes que debe alcanzar todo el conjunto del profesorado. Para Zambrano et al. (2005), la evaluación del desempeño profesional docente, se realiza a través de un cuestionario diseñado ad hoc, el Cuestionario de Evaluación del Desempeño Docente (en adelante CE$D E D$ ) que evalúa 11 dimensiones a través de 60 ítems. Las diferentes dimensiones están ajustadas a partir de los objetivos clave fijados para la evaluación. Existen estudios que analizan el desempeño profesional a partir de competencias, es decir, la resolución de problemas 
(Piminenta, 2014). Sin embargo, en numerosas ocasiones, la dificultad de la evaluación del contexto educativo no solo implica la evaluación del docente, sino la evaluación de los numerosos factores que influyen en el rendimiento académico del alumnado. Este hecho, se evidencia principalmente, a partir de los pobres resultados académicos obtenidos por los estudiantes. Por ello, en muchos de los países en los que se han realizado evaluaciones de la competencia docente entre el profesorado, el objetivo era aportar un diagnóstico de los aspectosque han podido condicionar el fracaso (M artínez, Castillo \& Granada, 2017; Pavié, 2011; Pimienta, 2014).

En cuanto a la evaluación de los agentes implicados, el desempeño profesional es evaluado no solo por parte del profesorado, sino también, por parte del alumnado. N ormalmente, se realiza a través de cuestionarios, con el objetivo de establecer una visión directa de las competencias que posee el profesorado vistas desde el propio al umnado (Arias deVega \& O Imos; Casero, 2008; Zambrano et al., 2005). En otras ocasiones, el desempeño profesional mide una competencia concreta, es el caso de la evaluación de la competencia digital, la cual establece el nivel de dominio de un sujeto en una herra mienta concreta. En la mayor parte de los estudios, la evaluación se realiza a través de cuestionarios ad hoc 0 diseñados específicamente para su propio contexto profesional (Agreda, Hinojo, \& Reche, 2016; GutiérrezCastillo, Cabrero-Almenara, \& Estrada-Vidal, 2016).

0 tro factor para destacar es que el desempeño profesional no es un constructo unidimensional, sino multidimensional. Algunos de los trabajos revisados que muestran escalas de evaluación, sitúan hasta once dimensiones (Zambrano et al., 2005). En nuestra propuesta, se diseñaun cuestionario propio a partir del cuestionario CEDED (Zambrano et al., 2005), del cual se han adaptado tres dimensiones: a) Interés por la forma ción continua, b) Formación enTIC y c) Autoevaluación. El motivo fundamental se debe a tres razones, la primera de ellas la naturaleza propia del contexto deportivo y especialmente el de los entrenadores, los cuales, deben estar actual izados en sus dominios y competencias profesionales, tales como las competencias tácticas, técnicas, físicas y psicológicas, por ello, se necesita una formación continua con el objetivo de estar actualizado en las cuestiones prioritarias en el cumplimiento de su rol (Lozano, Camerino, \& Hileno, 2015; Steele \& Vera, 2011; Vergara, Garbey, \& Fuentes, 2018). Por otro lado, no importa si se trata de niveles elevados de rendimiento, puesto que para García-N aveiray Jerez (2012), los entrenadores tienen entre sus funciones las de ase- sorar, orientar, formar, evaluar, intervenir e incluso investigar en las cuatro áreas de rendimiento citadas anteriormente. Además, aunque cuentan con la ayuda de especialistas (en el ámbito de alto rendimiento deportivo) 0 de colaboradores, que pueden actuar como ayudantes, el entrenador debe conocer las nuevas formas de trabajo de cada una de las situaciones de entrenamiento y competición, por ello se justifica una formación continua de lostécnicos. La segunda de las razones versa en la necesidad de una formación permanente en el dominio de lasTIC, puesto que en el fútbol actual, el control de las cargas y el análisis del rendimiento de los futbolistas se debe principalmente al uso y dominio de la tecnología. Destaca en este aspecto el dominio y lectura de valores ofrecidos por los GPS, cardiofrecuencímetros, acelerómetros (Buchheit, et al., 2012; Ėolakhodžiæet al. , 2016; Sañudo, 2017). Esinteresante destacar el papel de lasTIC no solo como herra mienta de control, sino como una herramienta válida para el desarrollo de la preparación física. Destaca en este sentido un estudio en el que se ha investigado el papel de lasTIC como herramienta eficaz para abordar trabajo no presencial en el entrenamiento, aspecto de especial interés tras la pandemia vivida en este año 2020 (Guillen, Herrera \& Ale de la Rosa, 2018). Finalmente, un técnico debe ser consciente de sus fortalezas y debilidades, las cuales, pueden orientar la buena dirección del conjunto de futbolistas, personal de apoyo y colaboradores. Por esta razón la autoevaluación resulta un contenido fundamental para valorar su desempeño profesional (Zambrano et al., 2005). Entre la literatura especializada revisada, existe una escasa productividad de investigaciones que se centran en la evaluación del desempeño profesional en el contexto deportivo y especialmente, entre los entrenadores de fútbol. De entre las propuestas que han sido pioneras en la evaluación del desempeño profesional de los técnicos, destacan los tra bajos de García-N aveira y J erez (2012), Martínez, Castillo y Granada (2017) y especialmente la deVergara et al. (2018) porque es aplicable al ámbito del fútbol, aunque en su caso, se trate de fútbol sala.

Finalmente, se deben destacar propuestas que han tenido por objetivo evaluar la formación de los entrenadores de fútbol base. Resultan muy interesantes los resultados obtenidos en el estudio de Paixão, Abad y Giménez (2019). En su estudio se concluye que su esca la es una herramienta válida para evaluar las posibles necesidades formativas de los técnicos deportivos.

A tendiendo puesatodos los principios anteriormente analizados, se señala el objetivo fundamental de esta 
propuesta como diseñar (adaptar) y validar una escala para evaluar el desempeño profesional de los entrenadores de fútbol en base a su formación permanente, nivel de TIC y autoevaluación. Para ello, se han revisado en la literatura especializada, escal as de valoración de contextos similares, como el educativo, justificado por la necesaria obligatoriedad de educar que deben poseer los nuevos entrenadores deportivos, tal y como exige la UEFA a partir de su convención celebrada en 2015. El estudio se ha realizado con entrenadores de fútbol en periodo de formación de la ComunidadValenciana durante el curso 2019-2020, con la finalidad de evaluar y valorar si la incorporación de innovaciones metodológicas en su formación actual y el aumento de Ios recursos en TIC ha permitido aumentar su desempeño profesional.

\section{Método}

\section{Participantes}

La muestra está formada por un total de 412 estudiantes de los cursos de formación de entrenadores de fútbol gestionados por la Federación de Fútbol de la Comunidad Valenciana (FFCV), bajo el amparo de la Real Federación Española de Fútbol (RFEF). Todos los entrenadores que forman parte de la presente investigación han realizado los cursos federativos normaliza dos tras la convención de la UEFA celebrada en 2015. En ella se realizaron las adaptaciones metodológicas pertinentes, adaptadas de forma similar al modelo educativo universitario, Espacio Europeo de Educación Superior (EEES). En cuanto a las características sociodemográficas, encontramos, en primer lugar, que respecto al género la muestra presenta una mayor proporción de hombres $(91.7 \%)$ que de mujeres (8.3\%). La edad media es de 30.88 (DT=10.50). Respecto al nivel de estudios, un $11.9 \%$ tienen estudios primarios, un $17 \%$ estudios secundarios, un $18.5 \%$ estudios de ba chiller, $17.5 \%$ estudios de ciclo formativo superior y un $35 \%$ estudios universitarios. Por último, según el nivel como entrenadores, un $71.1 \%$ tienen el título de M onitor UEFA C, un $4.6 \%$ el Nivel I de UEFA B, el $6.8 \%$ el Nivel II de UEFA A y el $17.5 \%$ el Nivel III de UEFA PRO.

\section{Instrumento}

Para diseñar el instrumento de medida, se utilizó el Cuestionario de Evaluación del Desempeño Docente (CEDED; Zambrano et al., 2005) que contenía60 ítems agrupadosen once factores: autoevaluación del alumno, cumplimiento del programa, dominio, calidad y pertinencia del profesor, utilización de material es didácticos, evaluación de las instalaciones, apoyo bibliográfico, asistenciay per manenciadel profesor, habilidades didácticas, calidad de los materiales, responsabilidad del alumno y habilidades de comunicación del alumno.

De los once factores se han seleccionado tres: asistenciay permanencia, utilización de materiales didácticos y autoevaluación. Estos factores pretenden evaluar tres áreas del desempeño profesional de los entrenadores de fútbol consultados: a) interés por la formación continua, que se integra el factor de asistencia y permanencia, b) formación en TIC, que se integra en el factor de utilización de materiales didácticos y c) autoevaluación, que se integra en el factor de autoevaluación.

A partir de las tres dimensiones que acogen a los tres dominios seleccionados, se han adaptado 11 indicadores en base a los ítems del cuestionario CEDED. Todos los indicadores han sido evaluados mediante una escala de respuesta tipo Likert ordenada de 1 a 5 de frecuencia: 1=nunca, 2=casi nunca, $3=$ alguna vez, $4=$ casi siempre y $5=$ siempre. Al final de la encuesta se ha creado un apartado para recoger la información sobre las características sociodemográficas de los participantes: edad, género, nivel como entrenadores y nivel de estudios.

\section{Procedimiento}

Desde marzo de 2019, la FFCV bajo la tutela de la Real Federación Española de Fútbol (RFEF) y ampara dos bajo la UEFA, inició los cursos UEFA C (antiguo curso de monitor deportivo en fútbol), así como el curso UEFA B (antiguo nivel I), dejando para el año 2020 Ios cursos UEFA A (antiguo nivel II) y el UEFA PRO (antiguo nivel III). Todos los cursos se han real izado en el territorio autonómico, Castellón, Valencia y Alicante.

Tras las pertinentes reuniones con la dirección territorial de la escuela de entrenadores de la Federación Valenciana, se firmaron las autorizaciones para realizar la investigación. El proceso, consistió en la administra ción de un cuestionario a través de un soporte on line (limesurvey) y de manera presencial en los días en los que se realizaban pruebas presenciales de evaluación. Los cuestionarios administrados de forma presencial se realizaron en las tres provincias de la comunidad autónoma. Las pruebas de evaluación presenciales se iniciaron el 1 de septiembre de 2019 y finalizaron el domingo 8 marzo de 2020, mientras que los cuestionarios on line, se han mantenido abiertos en la plataforma hasta el 15 de julio de 2020. 


\section{Análisis estadístico}

En primer lugar, se realizó un análisis factorial exploratorio (AFE), mediante el programa FACTOR a partir de las recomendaciones de Lloret-Segura, Ferreres-Traver, Hernández-Baeza y Tomás-M arco (2014). Este análisis se realizó mediante el método de extracción de MáximaVerosimilitud (MV) y se utilizó el método de rotación 0 blimin Directo. Para deter minar el número de factores se utilizó el procedimiento de 0 ptimización Implementada del Análisis Paralelo (Timmerman \& Lorenzo-Seva, 2011), mientrasque para comprobar el ajuste del modelo se analizaron los coeficientes de la raíz media cuadrática residual (RMCR) y el índice gamma o de bondad de ajuste (GFI) propuesto por Tanaka y Huba (1989). O tros indicadores que se tuvieron en cuenta fueron el índice Generalized G-H Index para analizar la replicabilidad de los factores derivados del AFE. También se observaron las medidas de adecuación muestral de Kaiser Meyer O Ikin (KMO) y el test de esfericidad de Barlett. Por otro lado, los ítems con cargas factoriales inferiores a .30 o superiores a este valor en dos o más factores se eliminaron antes de realizar el siguiente AFE. Por último, se comprobó la interpretabilidad teórica de la solución factorial extraída del AFE.

En segundo lugar, se realizó un anál isis factorial confirmatorio (AFC), aplicando el método robusto de estimación de máxima verosimilitud con la finalidad de corregir laposible ausenciade normalidad multivariante, mediante la utilización de estadísticos como el $x^{2}$ de Satorra Bentler (Chou, Bentler, \& Satorra, 1991). Así pues, para la evaluación del ajuste global, se hizo uso de distintos índices de bondad de ajuste recomendados en la literatura(K line, 2005), como lasignificación del Chicuadrado y de su corrección robusta proporcionada por Satorra-Bentler (S-B x²) (Satorra \& Bentler, 1994). También, se calcularon otros coeficientes que permitieron comprobar la adecuación de los modelos propuestos como el ratio de $x^{2}$ y sus grados de libertad $\left(x^{2} / \mathrm{gl}\right.$; Wheaton, Muthén, Alwin, \& Summers, 1977), siendo aceptables valores inferiores a cinco (Byrne, 2009). Asimismo, se comprobaron los coeficientes de los índices de bondad de ajuste robustos del modelo propuesto correspondiente al Índice de Ajuste Comparado (CFl; Bentler, 1990) y el Índice de Ajuste Incremental (IFI). Para estos indicadores se considera un buen ajuste los valores superiores a .90 (M acCallum \& Austin, 2000). Para finalizar, se muestra la raíz cuadrada media del error de aproximación (RM SEA), siendo necesario para considerar un buen ajuste del mismo puntuaciones infe- rioresa.08 (Browne \& Cudeck, 1993).

En la evaluación de la fiabilidad de la escal a se tuvieron en cuenta tres medidas: alfa de Cronbach, la Fiabilidad Compuesta (FC) y la M edida de laVarianza Extraída (AVE) para cada factor (Hair, Black, Babin, Anderson, \& Tatham, 2006). Por otro lado, también se comprobó la validez convergente a través de la significatividad de las cargas factoriales en su respectiva dimensión y los valores de las pruebas t asociados. Además, la validez discriminante, que tiene que ver con la distinción clara entre cualquier par de constructos, se evaluó mediante el método sugerido por Fornell y Larcker (1981). Este método admite la validez discriminante si la raíz cuadrada del valor AVE de un determinado factor es mayor que los coeficientes de correla ción entre el factor y cualquier otro factor de la escala propuesta. 0 tro criterio para asegurar la validez discriminante señala que las correlaciones entre los diversos pares de factores deben ser inferioresa. 85 (Kline, 2005).

\section{Resultados}

\section{Estadísticos descriptivos}

En la tabla 1 se muestran la media, desviación típica, asimetría y curtosis de cada indicador. Tal y como se puede observar la mayoría de los indicadores presentan valores superiores al valor 3 de la escala Likert, que indicaría una frecuencia reducida respecto a los aspectos evaluados por los entrenadores. Los indicadores con puntuaciones más reducidason los relacionados con «participo en foros, espacios de reflexión, redes de entrenadores que usan TIC en sus entrenamientos» ( $M=2.65$; $\mathrm{DT}=1.19$ ) y «acudo a los cursos de formación del entrenador que gestiona la Federación de Fútbol para actualizar mis conocimientos» ( $M=2.94 ; D T=1.21$ ), que indican una escasa frecuencia respecto a estas acciones formativas. En cuanto a los indicadores con una mayor puntuación destacan: «considero adecuado ver entrena mientos y partidos de rivales para mejorar mis competencias como técnico» ( $M=4.07 ; D T=.96)$ y «tengo mejores resultados deportivos gracias a mi actualización profesional» ( $M=3.98 ; \mathrm{DT}=.98)$, que indican una frecuencia elevada respecto a estas acciones. Por otro lado, los valores de asimetría y curtosis son aceptables ya que son inferiores a 3.0 en todos los ítems (Chou $\&$ Bentler, 1995).

\section{Análisis factorial exploratorio}

La validez interna de la escala de evaluación del desempeño profesional en técnicos de fútbol se contrastó a través de un análisis factorial exploratorio (AFE), a par- 
Tabla 1

Media, desviación típica, asimetría y curtosis de los indicadores de la escala de evaluación de desempeño profesional en técnicos de fútbol. Media AsymmetryKurtosis

espacios de formación y evaluacícíco en

2. Acudo a los cursos de formación del entrenador que gestiona la $2.94 \quad 1.21 \quad 00$ Federación de Fútbol para actualizar mis conocimientos.

3. Leo publicaciones actuales sobre metodologías de entrenamiento. $\begin{array}{llll}3.55 & 1.05 & -.41\end{array}$

4. Considero adecuado ver entrenamientos y partidos de rivales para $\begin{array}{cccc}4.07 & .96 & -.98\end{array}$

mejorar mis competencias como técnico.

5. Participo en foros, espacios de reflexión, redes de entrenadores $2.65 \quad 1.19 \quad .29$

que usanTIC en sus entrenamientos.

6. Continúo formándome en el manejo de herramientas TIC y su $3.25 \quad 1.10 \quad-.05$

$\begin{array}{lllll}\text { incorporación a mis responsabilidades como técnico. } & & & \\ \text { 7. Aprendo a usar herramientas y aplicaciones TIC de forma } 3.38 & 1.07 & -.29\end{array}$

7. Aprendo a usar herramientas y aplicaciones TIC de forma $3.38 \quad 1.07-2.29$

8. Reviso los programas de cursos para formarme e integrar las TIC $\begin{array}{llll}3.23 & 1.03 & -.20\end{array}$

en mi bagaje como entrenador.

9. Me considero mejor entrenador con el uso de TIC $\quad 3.47 \quad 1.09 \quad-.58$

10. 0 btengo mayor control del entrenamiento a través de lasTIC $\quad 3.57 \quad 1.03 \quad-.72$

11. Tengo mejores resultados deportivos gracias a mi actualización $3.98 \quad .98 \quad-1.01$ profesional

Nota: DT = Desviación típica; $1=$ nunca; $2=$ casi nunca; $3=$ alguna vez; $4=$ casi siempre; $5=$ siempre.

tir de Lloret-Segura et al. (2014) y, posteriormente, un análisisfactorial confirmatorio (AFC). Se realizó unAFE para los 11 ítems de la escala (ver tabla 2). El procedimiento de optimización implementada del análisis pa ralelo recomendaba la unidimensionalidad de la escala. No obstante, se decidió comprobar la solución factorial de tres factores debido a que permitía ofrecer una mejor interpretación teórica y permitía retener más indicadores en el AFC posterior para lograr un buen ajuste. Los resultados del AFE permitieron identificar tres factores en los que se agruparon los indicadores: interés por la formación continua (4 ítems), formación enTIC ( 3 ítems) y autoevaluación (4 ítems). Para comprobar el ajuste del modelo se analizaron los coeficientes de la raíz media cuadrática residual (RMCR) y el índice gamma o GFI, que mostraron coeficientes dentro de los puntos de corte recomendados: $\mathrm{RMCR}=.03(<.05)$ $\mathrm{GFI}=.99(>.95)$. Por otro lado, el Generalized G-H Index, mostró valores cercanos 0 superiores a .80 en todos los factores detectados por el AFE (oscilando en-

Tabla 2.

Estructura factorial rotada indicadores de la escala de evaluación del desempeño profesional en técnicos de fútbol, comunalidades, autovalores y varianza explicada.

\begin{tabular}{|c|c|c|c|}
\hline \multirow{2}{*}{\multicolumn{4}{|c|}{ Factor 1 - Interés por la formación continua }} \\
\hline & & & \\
\hline $\begin{array}{l}\text { 1. Participo en espacios de formación y evaluación sobre contenidos } \\
\text { específicos y novedosos en el ámbito del fútbol }\end{array}$ & .72 & & \\
\hline $\begin{array}{l}\text { 2. Acudo a los cursos de formación del entrenador que gestiona la } \\
\text { Federación de Fútbol para actualizar mis conocimientos. }\end{array}$ & .75 & & \\
\hline 3. Leo publicaciones actuales sobre metodologías de entrenamiento. & 41 & & \\
\hline $\begin{array}{l}\text { 5. Participo en foros, espacios de reflexión, redes de entrenadores que } \\
\text { usanTIC en sus entrenamientos. } \\
\text { Factor } 2 \text { - Formación en TIC }\end{array}$ & .37 & & \\
\hline $\begin{array}{l}\text { 6. Continúo formándome en el manejo de herramientas TIC y su } \\
\text { incorporación a mis responsabilidades como técnico. }\end{array}$ & & .75 & \\
\hline 7. Aprendo a usar her ramientas y aplicacionesTIC de for ma autónoma. & & .95 & \\
\hline $\begin{array}{l}\text { 8. Reviso los programas de cursos para formarme e integrar las TIC en } \\
\text { mi bagaje como entrenador. } \\
\text { Factor } 3 \text { - Autoevaluación }\end{array}$ & & .60 & \\
\hline $\begin{array}{l}\text { 4. Considero adecuado ver entrenamientos y partidos de rivales para } \\
\text { mejorar mis competencias como técnico. }\end{array}$ & & & .30 \\
\hline 9. Me considero mejor entrenador con el uso de TIC & & & .85 \\
\hline 10. O btengo mayor control del entrenamiento a través de lasTIC & & & .88 \\
\hline $\begin{array}{l}\text { 11. Tengo mejores resultados deportivos gracias a mi actualización } \\
\text { profesional }\end{array}$ & & & .54 \\
\hline G H Index & .79 & .90 & .89 \\
\hline Items & 4 & 3 & 4 \\
\hline
\end{tabular}

tre .79 y .90), señalando una buena replicabilidad de las dimensiones en otros estudios (Ferrando \& LorenzoSeva, 2017). Lavarianzaexplicada por los 11 ítemsagrupados en los tres factores fue del $67.51 \%$.

\section{Análisis factorial confirmatorio}

A partir de la solución factorial propuesta por el AFE se realizó un AFC, que mostró un buen ajuste, tal y como se puede observar en los índices de bondad de ajustedel modelo: chi-cuadrado significativo $\left(x^{2}=113.50\right.$, $g \mid=41, p<.01)$ y un valor del chi-cuadrado normado $\left(x^{2} / g l=2.77\right)$ inferior a $5 \mathrm{y}$ el índice RMSEA mostró un valor de .057 (Intervalo de confianza=.042-.072), inferior a .08. En la misma línea, el resto de los índices muestran un buen ajuste del modelo, ya que presenta ban valores superiores a $.96: \mathrm{CF}=.97 ; \mathrm{e} \mid \mathrm{Fl}=.97$.

Para analizar la fiabilidad se observaron las medidas del alfa de Cronbach, la fiabilidad compuesta (FC), y la Varianza M edia Extraída (AVE), cuyos valores se ajustaron a los parámetros recomendados por la literatura: Formación TIC ( $\alpha=.76 ; F C=.76 ; A V E=.44)$, interés por la formación continua (á=.89; $\mathrm{FC}=.89 ; \mathrm{AVE}=.72$ ) y autoevaluación ( $\alpha=.77 ; F C=.78 ; A V E=.50)$. En el caso de la dimensión de desarrollo de la docencia se observó un valor del AVE ligeramente inferior a .50 recomendado por la literatura. De acuerdo con Hatcher (1994), cuando la fiabilidad del constructo es aceptable, se puede aceptar un valor marginalmente bajo del AVE (ver tabla 3). En este caso, los valores de fiabilidad compuesta fueron superiores a 70 que son valores aceptables de acuerdo con la literatura (Hair et al., 2006).

Se comprobó que los valores de las pruebast asocia dos a las cargas factoriales de los ítems eran superiores a $1.96(p<.05)$, oscilando desde 5.26 hasta 13.48 , permitiendo comprobar la validez convergente. En cuanto

Tabla 3

Cargas factoriales, fiabilidad compuesta, medida de la varianza extraída y alfa de Cronbach de los indicadores de la escala de evaluación del desempeño profesional en técnicos de fútbol.

\begin{tabular}{lrrrr}
\hline & $\lambda$ & $\alpha$ & FC & AVE \\
\hline Factor 1 - Interés por la formación continua & .76 & .76 & .44
\end{tabular}

1. Participo en espacios de formación y evaluación sobre contenidos .74 específicos y novedosos en el ámbito del fútbol

2. Acudo a los cursos de formación del entrenador que gestiona la .64

Federación de Fútbol para actualizar mis conocimientos.

3. Leo publicaciones actuales sobre metodologías de entrenamiento. .59

5. Participo en foros, espacios de reflexión, redes de entrenadores que .68

usan TIC en sus entrenamientos.

Factor 2 - Formación en TIC

6. Continúo formándome en el manejo de herramientas TIC y su .88

incorporación a mis responsabilidades como técnico.

7. Aprendo a usar herramientas y aplicacionesTIC de forma autónoma. .83

8. Reviso los programas de cursos para formarme e integrar las TIC en

mi bagaje como entrenador.

Factor 3 - Autoeval uación

4. Considero adecuado ver entrenamientos y partidos de rivales para .34

mejorar mis competencias como técnico.

9. Me considero mejor entrenador con el uso deTIC

10. O btengo mayor control del entrenamiento a través de lasTIC

11. Tengo mejores resultados deportivos gracias a mi actualización 63 profesional $\begin{array}{lll}.89 & .89 & .72\end{array}$

$.77 \quad .78 \quad .50$

.72


a la validez discriminante, por un lado, comprobamos que todas las correlaciones entre los diversos factores fueron inferiores a .85, cumpliéndose dicho criterio como se puede observar en la tabla 5. Por otro lado, se comprobó que la raíz cuadrada del AVE era superior a la correlación entre pares de factores, este criterio únicamente no se cumplió en el caso de la correlación entre los factores 1 y 3 (ver tabla 4).

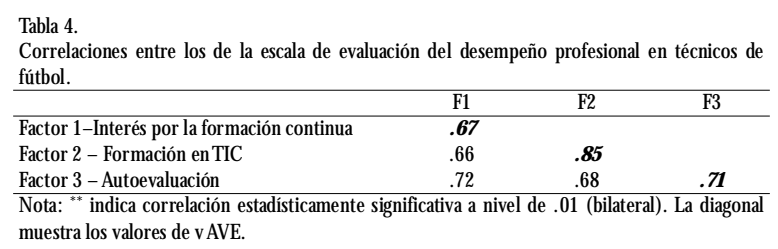

\section{Discusión}

A partir de los resultados obtenidos en la investiga ción, se demuestra que la escala de evaluación del desempeño profesional de los entrenadores de fútbol presenta unas propiedades psicométricas adecuadas para medir este constructo. Los tres factores que integran la escala muestran una alta consistencia interna tal como sugieren Hair et al. (2006) a partir de los valores para un ajuste adecuado. Por otro lado, se cumplen los criterios especificados por la literatura para asegurar la val idez convergente y discriminante, que sugieren que el instrumento utilizado cumple con las exigencias que se requieren para evaluar el desempeño profesional.

Por otro lado, los tres factores muestran índices adecuados de fiabilidad de acuerdo con las especificaciones de la literatura, con valores superiores a .70 para los tres factores propuestos. Estos resultados van en la misma línea que los obtenidos por Zambrano et al. (2005) que presentan en su cuestionario (CEDED) un valor en el Alfa de Cronbach de .97.

Al igual que el trabajo de Pimienta (2014), encontramos para la evaluación del desempeño profesional tres factores. En la línea que argumenta el autor, y tras el análisis factorial exploratorio, se obtuvieron 11 ítems de la escala, para ello y tras comprobar su adecuación a cada factor, se decidió ver la solución factorial. En el AFC posterior, el objetivo se centró en comprobar y lograr un buen ajuste. Así pues, los resultados del AFE permitieron identificar tres factores en los que se agruparon los indicadores: interés por la formación continua (4 ítems), formación en TIC (3 ítems) y autoevaluación (4 ítems).

En cuanto a las reflexiones generadas a partir de conclusiones de otras investigaciones, en nuestra pro- puesta, se muestra una línea similar a la que ofrece el trabajo propuesto por Rodríguez, Lara y Rodríguez (2018), en la que, según los autores, existen muchas limitaciones para realizar evaluaciones a los técnicos, especialmente en la iniciación deportiva. También transitan en esta línea de evidenciar las limitaciones existentes en la evaluación del desempeño profesional, el estudio realizado por Vergara et al. (2018), en el que se destaca la afirmación de que no existe un criterio de objetividad para poder evaluar el desempeño profesional de los técnicos de fútbol sala.

En el análisis de escalas que evalúan una de las competencias profesionales, que está en la línea propuesta en este trabajo, la evaluación de la formación en TIC, destaca la propuesta realizada por Gutiérrez-Castillo et al. (2016). En su propuesta, destaca que cada vez es más evidente en la sociedad actual el poseer un dominio competencial de las TIC, además, en su escala de evaluación se justifica que pueda existir una escala objetiva que evalúe la competencia digital. La escala del estudio de la propuesta de Gutiérrez-Castillo et al. (2016) presenta un Alfa de Cronbach con un valor de .96, por lo que muestra valores muy adecuados para valorar su validez. En esta línea de resultados, la presente escala se muestra en este sentido similar a los resulttados encontrados por Gutiérrez-C astillo et al. (2016), con un valor en el Alfa de Cronbach para el factor de formación en TIC de 0.89.

Respecto a otras escalas que evalúan el desempeño profesional, como la realizada por Casero (2008), se muestran una reducción de los factores de 11 a 3. Este hecho muestra el carácter multidimensional de la escala, que como se ha comentado, está constituida por tres dimensiones. El resultado obtenido por la investigación realizada, cumple y satisface los objetivos planteados en la propuesta, además, ofrece una herramienta de evaluación del desempeño profesional, lo cual sugiere que está de acuerdo con los resultados obtenidos por el estudio realizado por Casero (2008).

El resultado de la escala diseñada a partir del cuestionario de Zambrano et al. (2005) muestra, en primer lugar, que se ha obtenido una herramienta válida y fia ble, lo cual confirma que se ha cumplido el objetivo principal de la investigación. Por otro lado, el cuestionario, queda al servicio de las instituciones deportivas como las Federaciones y clubes deportivos para tener una herramienta válida en la evaluación del desempeño profesional de los entrenadores sin tener como criterio único los resultados deportivos.

Por otro lado, debe indicarse, que una de las limita 
ciones que presenta la escala consiste en que no se han evaluado entrenadores con formación por otras vías diferentes a la federativa, aspecto que puede generar una futura investigación, teniendo como población de estudio, entrenadores formados por la vía autonómica.

Se debe destacar, que la muestra no es representativa del total de los entrenadores de la ComunidadValenciana. Este hecho se justifica porque no se ha selecciona do a ningún técnico formado por la vía de Conselleria de Educación. Finalmente, destacar el hecho de haber empleado dos vías de recopilación de datos, la presencial y la on line.

En cualquier caso, se debe seguir comprobando el funcionamiento de la escala en otras muestras de entrenadores con el objetivo de confirmar el adecuado funcionamiento de la herramienta para evaluar el desempeño profesional de los técnicos, así como la independencia entre el factor de interés por la formación continua y el factor de autoevaluación. Del mismo modo, en futuros estudios, se debe revisar las propiedades del ítem número cuatro ya que su carga factorial en este trabajo es bastante reducida (.34) y podría tener una mayor relación con el factor de interés por la formación continua en lugar del factor de autoevaluación. En este tra bajo, se comprobó que el ajuste de la escala en el AFC no mejoraba significativamente al eliminar este indica dor de la escala e incluso empeoraba los índices de ajuste al trasladarlo a la dimensión de interés por la forma ción continua, mostrando una carga factorial más reducida cuando se incorporaba a este factor (.31). Por esta razón, se decidió mantener el indicador en la dimensión de autoevaluación.

Como futura propuesta de investigación se podrían establecer comparaciones en la formación permanente, el dominio de lasTIC y la autoevaluación que poseen los técnicos formados por la línea académica no federativa. Este aspecto establecería una línea paralela de la formación de los entrenadores y no limitar a ninguna de las opciones elegidas por los entrenadores.

\section{Conclusiones}

El cuestionario, Escala para la evaluación del desempeño profesional del entrenador de fútbol en base a su formación permanente, nivel de TIC y autoevaluación, ha demostrado ser válida y fiable para la evaluación del desempeño profesional de los técnicos deportivos, en este caso entrenadores de fútbol. Se comprobó que la escala presentaba propiedades psicométricas adecuadas, comprobando su validez a través de los resultados de los análisis factoriales y la fiabilidad a través del alfa de Cronbach, la fiabilidad compuesta y la varianza media extraída.

La escala es de carácter multidimensional confirmando la presencia de tres dimensiones o áreas que integran el constructo de evaluación del desempeño profesional del entrenador de fútbol: interés por la formación continua ( 4 ítems), formación en TIC ( 3 ítems) y autoevaluación (4 ítems).

El estudio del desempeño profesional entre el colectivo de los entrenadores de fútbol es fundamental para investigar las relaciones que se puedan establecer entre la formación de los técnicos y los resultados deportivos obtenidos. Además de la importancia de estar continuamente actualizado en los contenidos de rendimiento asociados al fútbol, como son los contenidos de tipo táctico, técnico, físico y psicológico, lasTIC ofrecen a partir de herramientas especializadas, el control de cargas, la valoración del rendimiento de los deportistas, el almacenamiento de datos, entre otros muchos aspectos. Este aspecto evidencia, que los entrenadores formados por las federaciones territoriales, bajo el control de la RFEF y la UEFA, deben ser conocedores de las herramientas adecuadas a su nivel de competición. Por último, la labor evaluativa en per sonas de contextos de rendimiento, como son los entrenadores de fútbol, es fundamental para realizar lecturas adecuadas de los procesos de competición. LaUEFA, ha sabido actuar en consecuencia adaptando la formación de los técnicos a los nuevos espacios europeos de educación, como anteriormente realizaron las U niversidades Europeas.

\section{Referencias}

Agreda, M., Hinojo, M. A ., \& Reche, J. M. (2016). Diseño y validación de un instrumento paraevaluar lacompetenciadigital de los docentes de la educación superior española. Píxel-Bit, Revista de Medios y Educación, 49, 39-56. https:/ / doi.org/ 10.12795/ pixelbit.2016.i49.03

AriasdeVega, E., \& O ImosGómez, M. del C. (2015). Evaluación del desempeño profesional deformadores de docentesen el Salvador. Publicaciones, 45, 27-52.

Bentler, P. M. (1990). Comparative Fit Indexesin Structural M odel. Psychological Bull letin, 107(2), 238-246.

Browne, M.W. \& \& Cudeck, R. (1993). Alternativeways of assessing model fit.Testing structural equation models. KA Bollen and JSLong.

Buchheit, M., Simpson, M. B. , Al Haddad, H., Bourdon, P. C., \& Mendez-Villanueva, A. (2012). M onitoring changes in physical performance with heart rate measures in young soccer players. European Journal OfApplied Physiology, 112(2), 711-723. https: / / doi.org/ 10.1007/ s00421-011-2014-0

Byrne, B. M. (2009). Structural equation modeling with amos: Basic concepts, applications, and programing (2nd ed.). N ewYork: Routledge.

Casero, A. (2008). Propuestade un cuestionario de evaluación dela 
calidad docente universitaria consensuado entre alumnosy profesores. Revista delnvestigación Educativa, 26(1), 25-44.

Chou, C.-P., \& Bentler, P. M. (1995). Estimates and testsin structural equation modeling EnStructural equation modeling: Concepts, issues, and applications (pp. 37-55). Sage Publications, Inc.

Chou, C.-P., Bentler, P. M ., \& Satorra, A. (1991). Scaled test statistics and robust standard errors for non-normal data in covariance structure analysis: A Monte Carlo study. British Journal of M athematical and Statistical Psychology, 44 (2), 347-357. https:/ / doi.org/ 10.1111/ j.2044-8317.1991.tb00966.x

Èolakhodžiæ, E., Rað̃o, I., Skender, N., Kapo, S. \& Rašidagiæ, F. (2016). Differences in the D evelopmental Rates of Ventilation CapabilitiesBetween BoysW ho Play Football and BoysW ho Do N ot Play Football. H omo Sporticus, 18 (1), 7-13.

Escudero,T. (2019). Evaluación del Profesorado como camino directo hacialamejorade laCalidad Educativa. Revista de Investigación Educativa, 37 (1), 15-37. https:// doi.org/ 10.6018/ rie. 37.1 .342521

Ferrando, P. J. , \& Lorenzo-Seva, U. (2017). Program FACTO R at 10: 0 rigins, development and future directions. Psicothema, 29.2, 236240. https: / / doi.org/ 10.7334/ psicothema2016.304

Fornell, C., \& Larcker, D. (1981). Structural equation models with unobservable variablesand measurement error:Algebra and statistics. (N. ${ }^{\circ}$ 266). The University of Michigan. https:/ / deepblue.lib. umich. edu/ bitstream/ handle/ 2027.42/ 35622/ b1378752.0001.001. pdf?sequence $=2$

Fraile-Aranda, A. , deDiego-Vallejo, R ., \& Boadai Grau, J. (2011). El perfil delostécnicos del deporteescolar en un contexto europeo. Revista Internacional de Medicina y Ciencias dela Actividad Física y del Deporte, 11(42), 278-297.

García-N aveira, A., \& Jerez, P. (2012). D epartamento de psicología del clubAtlético de Madrid: Filosofía, programación y desempeño profesional en el fútbol base. Cuadernos de Psicología del D eporte, 12(1), 11-120.

Guillen, L., Herrera, A., \&Ale delaRosa,Y. (2018). Lasherramientas tecnológicasT IC 'scomo elemento al ternativo parael desarrollo del componentefísico. Retos: N uevas Per spectivas de Educación Física, Deportey Recreación, 34, 222-229.

González, G., Campos, M., \& Romero, S. (2014). Análisis de la influencia de la evaluación del rendimiento en jugadores de un equipo de fútbol. Retos: N uevas PerspectivasdeEducación Física, D eportey Recreación, 25, 85-89.

Gutiérrez-Castillo, J., Cabrero-A Imenara, J. , \& Estrada-Vidal, L. I. (2016). Diseño y validación de un instrumento deeval uación de lacompetenciadigital del estudiante universitario. Espacios, 38(10).

Hair, J. F., Black, W. C., Babin, B. J., Anderson, R. E., \&Tatham, R. L. (2006). Multivariatedata analysis. New Jersey: Prentice Hall.

Hatcher, L. (1999). A step-by-step approach to using the SAS system for factor analysis and structural equation modeling. SASInstitute Inc., Cary, NC.

Kline, R. B. (2015). Principles and Practice of Structural Equation M odeling, Fourth Edition. NewYork: Guilford Publications.

Lledó, E. , M artínez, G. \& \& Huertas, F. (2014). Profile of a football coach for the school yearsin top clubs' academies in theValencian Community. Cultura, Ciencia y Deporte, 9(25), 57-68. https:/ / doi. org/ 10.12800/ ccd.v9i25.389

Lloret-Segura, S. , Ferreres-Traver, A., Hernández-Baeza, A., \&Tomás-M arco, I. (2014). El análisis factorial exploratorio de los ítems: Una guía práctica, revisaday actualizada. Anales dePsicología I Annals of Psychology, 30(3), 1151-1169. https:/ / doi.org/
10.6018/ analesps. 30.3.199361

Lozano, D., Camerino, O., \& Hileno, R. (2016). Analysis of the offensivetactical behavior in critical moments of gamein thehigh performance in handball:A study M ixed M ethods. Cuadernosde Psicología del Deporte, 16(1), 151-160.

MacCallum, R., \&Austin, J. (2000). Applications of Structural Equation Modeling in Psychological Research | Annual Review of Psychology. https: / / w w w. annual review s. org/ doi/ abs/ 10.1146/ annurev.psych. 51.1.201?journalCode=psych

Marcelo, C. (2011). Evaluación del desarrollo profesional docente. BarceIona: D avinci Continental. https: / / dial net. unirioja. es/ servlet/ libro?codigo $=742182$

Martínez, J., Castillo, L., \& Granada, V. (2017). Formación inicial del docentedeeducación físicay su desempeño profesional. R evista Digital de Educación Física, 8, 48.

Paixão, P. , A bad, M ., \& Giménez, F. (2019). Diseño y validación de un cuestionario para estudiar la formación de entrenadores de fútbol base. Retos: N uevas Perspectivas de Educación Física, Deportey Recreación, 35, 294-300.

Pavié, Á. (2011). Formación docente: Haciauna definición del concepto de competencia profesional docente. Revista Electrónica Interuniversitaria de Formación del Profesorado, 14(1), 67-80.

Pimienta, J. (2014). Elaboración y validación de un instrumento para la medición del desempeño docente basado en competencias. Revista de Docencia U niversitaria, 12(2), 231-250.

Rodríguez, H. , Lara, D., \& Rodríguez, C. (2018). El profesor en la iniciación deportiva y la evaluación de su desempeño. Lecturas: Educación Físicay D eportes, 22(237), 10.

Rojas, F. J., Cepero, M. , Zurita, F., \& Chinchila, J. L. (2015). Simulación del desempeño profesional, recurso didáctico en docencia deciencias del deporte. Revista Internacional deM edicina y Cienciasde laActividad Física y del D eporte, 15(57), 17-28.

Sañudo, B. (2017). N uevastecnologíasaplicadasa la actividad física y el deporte. Pamplona:Thomson ReutersA ranzadi.

Steele, S., \&Vera, S. (2011). Formación permanenteen el áreaEducación Física dirigidaal docente de educación integral. ARJÉ Revista dePostgrado FACE-UC., 5(9), 11-33.

Tanaka, J. S., \& Huba, G. J. (1989). A general coefficient of determination for covariance structure modelsunder arbitrary GLS estimation. British Journal of Mathematical and Statistical Psychology, 42 (2), 233-239. https:/ / doi. org/ 10.1111/ j.20448317.1989.tb00912.x

Timmerman, M. E., \& Lorenzo-Seva, U. (2011). Dimensionality assessment of ordered polytomousitems with parallel analysis. Psychological M ethods, 16(2), 209-220. https: / doi.org/ 10.1037/ a0023353

UEFA. (2015). Convención de la U EFA sobretitulacionestécnicas.

Vergara, M. , Garbey, A., \& Fuentes, N. (2018). Estrategiade supera ción parael mejorar el desempeño profesional del entrenador de futbol sala. Ciencia y Actividad Física, 5 (2), 94-106.

Wheaton, B. , Muthén, B. Alwin, D. F. \& Summers, G. F. (1977). Assessing Reliability and Stability in Panel M odels. Sociological Methodology, 8, 84-136. https:/ / doi.org/ 10.2307/ 270754

Zambrano, R., M eda, R. M., \& Lara, B. (2005). Evaluación de profesoresuniversitarios por parte delosalumnosmedianteel Cuestionario deEvaluación de D esempeño Docente (CEDED). Educación y D esarrollo, 4, 64-69. 